[knowledge base]

Diamond Open Access

\title{
Vector space over a field
}

\author{
Open Mathematics Collaboration*†
}

March 23, 2021

\begin{abstract}
VECTOR SPACE OVER A FIELD and its underlying definitions are presented in this white paper (knowledge base).
\end{abstract}

keywords: vector space, field, abstract algebra, knowledge base

The most updated version of this white paper is available at https://osf .io/g6ux5/download

Open Mathematics Knowledge Base http: //omkb.org

*All authors with their affiliations appear at the end of this white paper.

†Corresponding author: mplobo@uft.edu.br|Open Mathematics Collaboration 


\section{Definition}

\section{Vector Space over a Field (F-vector space)}

$$
(V, F,+, \cdot)
$$
(a) $(V,+):=$ commutative group
(b) Closure under (left) scalar multiplication: $\forall k \in F, \quad \forall v \in V, \quad k v \in V$
(c) Scalar (left) multiplication identity: $(1 \in F, v \in V) \rightarrow(1 v=v)$
(d) Associativity of (left) scalar multiplication:
$\forall j, k \in F, \quad v \in V, \quad(j k) v=j(k v)$
(e) (left) Distributivity of 1 scalar over 2 vectors:
$\forall k \in F, v, w \in V, \quad k(v+w)=k v+k w$
(f) (left) Distributivity of 2 scalars over 1 vector:
$\forall j, k \in F, \quad v \in V,(j+k) v=j v+k v$
$V, F:=$ sets
$(F,+, \cdot):=$ field
$+, \cdot:=$ binary operations on $V, F$
. $F \times V \rightarrow V$
(scalar multiplication between elements of $F$ and $V$ )
$[1,2]$ 


\section{Prerequisites}

\section{Field}

$$
(F,+, \cdot)
$$

(a) $(F,+):=$ commutative group

(b) $\left(F^{*}, \cdot\right):=$ commutative group

(c) Multiplication is distributive over addition in $F$

(d) $0 \neq 1$

$F:=$ set,$\quad F^{*}=F \backslash\{0\}$

$+, \cdot:=$ binary operations on $F$ (addition and multiplication)

$0:=$ additive identity, $\quad 1:=$ multiplicative identity

$[1,2]$

\section{Group}

$$
(G, \star)
$$

(a) Associativity: $\forall x, y, z \in G,(x \star y) \star z=x \star(y \star z)$

(b) Identity: $\exists e \in G: \forall x \in G, e \star x=x \star e=x$

(c) Inverse: $\forall x \in G \exists y \in G: x \star y=y \star x=e$

$G:=$ set

$\star:=$ binary operation

[1]

4. Commutative group (Abelian)

$$
\begin{gathered}
G_{b} \\
\forall g_{1}, g_{2} \in G_{b}, g_{1} g_{2}=g_{2} g_{1}
\end{gathered}
$$$$
G_{b}:=\text { group }
$$ 
$[2]$

5. Distributive

$$
\begin{array}{rr}
\forall x, y, z \in R: x \cdot(y+z)=x \cdot y+x \cdot z & \text { left distributive } \\
\forall x, y, z \in R:(y+z) \cdot x=y \cdot x+z \cdot x & \text { right distributive } \\
{[1,2,4]} &
\end{array}
$$

6. Binary operation

$$
\star: S \times S \rightarrow S
$$

$S:=$ set

$S \times S:=$ Cartesian product

[1]

\section{Cartesian product}

$$
A \times B=\{(a, b) \mid a \in A, b \in B\}
$$

$A, B:=$ sets

$A \times B:=$ Cartesian product

$(a, b):=$ ordered pair

[3]

8. Ordered pair

$$
(a, b)=\{\{a\},\{a, b\}\}
$$

$a:=$ first coordinate

$b:=$ second coordinate

$[1,3]$ 


\section{Open Invitation}

Review, add content, and co-author this white white paper [5,6]. Join the Open Mathematics Collaboration.

Send your contribution to mplobo@uft.edu.br.

\section{Open Science}

The latex file for this white paper together with other supplementary files are available in [7].

\section{Ethical conduct of research}

This original work was pre-registered under the OSF Preprints [8], please cite it accordingly [9]. This will ensure that researches are conducted with integrity and intellectual honesty at all times and by all means.

\section{Acknowledgements}

+ Center for Open Science https://cos.io

+ Open Science Framework https://osf.io

\section{References}

[1] Warner, Steve. Abstract Algebra for Beginners. GET 800, 2018. https://books.google.com/books?id=UFleyAEACAAJ 
[2] Dummit, David Steven, and Richard M. Foote. Abstract Algebra. Vol. 3. Hoboken: Wiley, 2004.

https://books . google.com/books?id=znzJygAACAAJ

[3] Velleman, Daniel J. How to prove it: A structured approach. Cambridge University Press, 2019.

https://books . google.com/books?vid=ISBN0521861241

[4] Cain, Alan J. Nine Chapters on the Semigroup Art. AJC Porto \& Lisbon, 2020. http://www-groups.mcs.st-and.ac.uk/alanc

[5] Lobo, Matheus P. "Microarticles." OSF Preprints, 28 Oct. 2019. https://doi.org/10.31219/osf.io/ejrct

[6] Lobo, Matheus P. "Simple Guidelines for Authors: Open Journal of Mathematics and Physics." OSF Preprints, 15 Nov. 2019.

https://doi.org/10.31219/osf .io/fk836

[7] Lobo, Matheus P. "Open Journal of Mathematics and Physics (OJMP)." OSF, 21 Apr. 2020.

https://doi.org/10.17605/osf .io/6hzyp

https://osf.io/6hzyp/files

[8] COS. Open Science Framework. https://osf .io

[9] Lobo, Matheus P. "Vector Space over a Field." OSF Preprints, 14 Mar. 2021. https://doi.org/10.31219/osf .io/g6ux5

\section{The Open Mathematics Collaboration}

Matheus Pereira Lobo (lead author, mplobo@uft.edu.br) $)^{1,2}$ https://orcid.org/0000-0003-4554-1372

${ }^{1}$ Federal University of Tocantins (Brazil)

${ }^{2}$ Universidade Aberta (UAb, Portugal) 